\title{
Ion channeling in T-phase quasicrystals: an investigation by Rutherford backscattering and particle induced X-ray emission
}

\author{
H.D. Carstanjen ${ }^{\text {a,b }}$, R.M. Emrick ${ }^{a, 1}$, T. Kupke ${ }^{b}$, D. Plachke ${ }^{a}$, R. Wittmann ${ }^{c}$ \\ and H.-R. Trebin ${ }^{b}$ \\ ${ }^{a}$ Max-Planck-Institut für Metallforschung, Institut für Physik, D-7000 Stuttgart 80, Germany \\ ${ }^{b}$ Institut für Theoretische und Angewandte Physik, Universität Stuttgart, D-7000 Stuttgart 80, Germany \\ 'Institut für Festkörperforschung, Forschungszentrum Jülich, D-5170 Jülich, Germany
}

The channeling properties of a $T$-phase $\mathrm{Al}_{62} \mathrm{Cu}_{20} \mathrm{Co}_{15} \mathrm{Si}_{3}$ quasicrystal are investigated in a Rutherford backscattering study employing $2 \mathrm{MeV}$ He-ions. Besides axial channeling along the decagonal axis, also planar channeling is observed in planes having the decagonal axis in common and showing the decagonal symmetry of the quasicrystal. Besides a system of main planes, various planes exhibiting only weak channeling properties are observed; they correspond to linear arrangements of vertices in a two-dimensional Penrose grid, demonstrating the close relationship between T-phase quasicrystals and Penrose pattern. Rutherford backscattering (RBS) has also been used to study the decoration of the T-phase structure by $\mathrm{Co} / \mathrm{Cu}$ - and $\mathrm{Al} / \mathrm{Si}$-atoms; in addition, particle induced X-ray emission PIXE served to distinguish between $\mathrm{Co}$ - and $\mathrm{Cu}$-atoms. The experimental data are compared with channeling computer simulations on a model T-phase quasicrystal proposed by Steurer and Kuo [ $[$ ].

\section{Introduction}

Up to the present time, many binary and ternary alloys have been synthesized which exhibit axes of fiveor tenfold symmetry in X-ray or transmission-electron diffraction pattern. Since an axis of five- or tenfold symmetry is strictly forbidden in ordinary periodic structures, many suggestions have been made to explain the observed diffraction pattern, such as twinned periodic crystals [1], randomly stacked oriented icosahedral clusters of atoms [2] and others. Perhaps the most striking was the concept of icosahedral Amman tiling of space [3] (a three-dimensional generalization of the two-dimensional Penrose pattern [4]), since it proposed a completely new class of crystalline structures, the so-called icosahedral quasicrystals.

In contrast to ordinary crystals which can be generated by the translation of one elementary cell and, hence, are periodic, icosahedral quasicrystals consist of two different elementary cells, a prolate and an oblate rhombohedron, which are stacked according to certain rules and fill space densely. The resulting structures have icosahedral symmetry with several five-, threeand twofold axes and, hence, are not periodic; they are

\footnotetext{
1 On leave from the Department of Physics, University of Arizona, Tucson, Arizona, USA.
}

called quasiperiodic. A special type of quasicrystals which we will be dealing with in this paper - are T-phase quasicrystals. They are periodic along one axis, but quasiperiodic in the plane perpendicular to this axis.

During the last few years, more evidence has been collected for the existence of the quasicrystalline structure. In particular the detailed analysis of X-ray diffraction patterns yielded strong support for this model; we mention in particular the thorough work of Steurer [5] and Steurer and Kuo [6] who used the method of high-dimensional embedding to derive electron density distributions and the positions of the individual atoms from $\mathrm{X}$-ray diffraction patterns.

Among the various experimental methods known to provide information on the structural properties of a crystal, fast ion channeling has not been used until very recently for the investigation of quasicrystals. In contrast to the diffraction methods, ion channeling is able to give direct information about the structure of a crystal in real space; however channeling presupposes the existence of linear and planar arrangements of the atoms in a crystal. Until very recently it was unknown whether such atomic strings or planes also exist in a quasicrystal. In a computer study on icosahedral quasicrystals Kupke et al. [7] showed (i) that the atoms of a quasicrystal also form strings and planes - in contrast to ordinary crystals the occupation of these strings and 
planes by atoms is not uniform - and (ii) that fast ion channeling should be feasible in quasicrystals. So far, only the experimental realization was missing.

\section{Experimental}

In the present investigation we show that ion channeling is possible also in quasicrystalline structures (a preliminary report on this subject is given in ref. [8]) \#! For this purpose a $\mathrm{T}$-phase $\mathrm{Al}_{62} \mathrm{Cu}_{20} \mathrm{Co}_{15} \mathrm{Si}_{3}$ quasicrystal of dimensions $0.8 \times 0.8 \times 0.8 \mathrm{~mm}^{3}$ was used which had been grown by one of us (R.W.) at the crystal laboratory of the Institut für Festkörperforschung at the Forschungszentrum Jülich. A short description of the growing procedure is given in the following; a more detailed description is found in ref. [9]. An alloy of nominal composition $\mathrm{Al}_{62} \mathrm{Cu}_{20} \mathrm{Co}_{15} \mathrm{Si}_{3}$ was prepared by induction melting of the high purity elements (at least $99.99 \%$ ) in a cold crucible under an argon atmosphere. Then the ingot was remelted in an aluminium oxide crucible and cooled from 1100 to $900^{\circ} \mathrm{C}$ at a rate of $10^{\circ} \mathrm{C} / \mathrm{min}$, followed by air-cooling down to room temperature. The ingot was crushed and one of the largest crystallites of decaprismatic shape selected for the experiments.

For the actual channeling experiment the crystal was mounted on a carbon backing in order to avoid interference of the scattering by the target support with the backscattering from the $\mathrm{Al}, \mathrm{Si}, \mathrm{Co}$ and $\mathrm{Cu}$ atoms of the quasicrystal. The RBS measurements were performed using a beam of $2 \mathrm{MeV}{ }^{4} \mathrm{He}^{+}$-ions, while for the PIXE measurements a $3 \mathrm{MeV}^{4} \mathrm{He}^{+}$-beam was used (beam size at the target: $0.5 \times 0.5 \mathrm{~mm}^{2}$, angular spread of the ion beam: $\pm 0.05^{\circ}$ ). For the recording of the channeling profiles, the backscattering rate of these ions and also in some cases the $\mathrm{X}$-ray yield was monitored during angular scans across several axes and planes of the quasicrystal.

\section{Results and discussion}

Fig. 1 shows typical backscattering spectra as obtained from this quasicrystal for ion incidence along a "random" direction, i.e. an arbitrary direction, and along the decagonal axis. For the recording of the $\mathrm{Co} / \mathrm{Cu}$ - and $\mathrm{Al} / \mathrm{Si}$-yield, energy windows were set on

"1 Part of these data were presented at the Spring Meeting of the German Physical Society, Münster, Germany, April, 1991 [13]. A study on channeling in icosahedral quasicrystals was presented at the 10th Int. Conf. on Ion Beam Analysis, Eindhoven, Netherlands, July, 1991 by Du Marchie van Voorthuysen et al. [14] as a postdeadline paper.

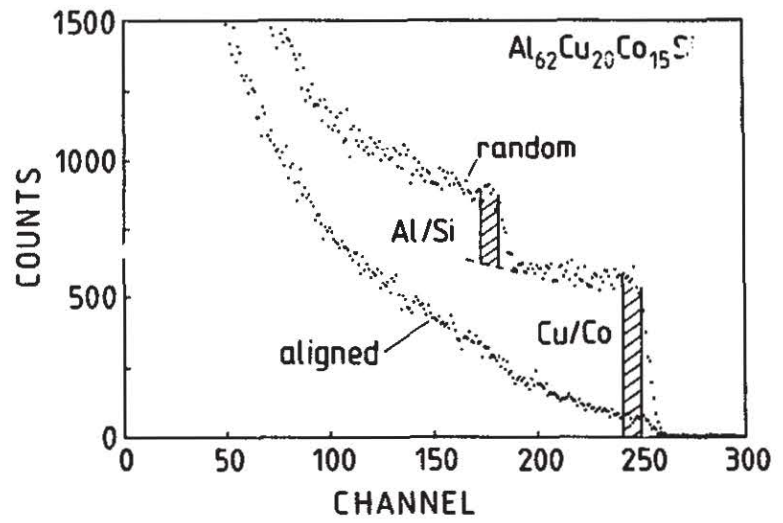

Fig. 1. Rutherford backscattering spectra of $2 \mathrm{MeV}$ He-ions as obtained from a $\mathrm{T}$-phase $\mathrm{Al}_{62} \mathrm{Cu}_{20} \mathrm{Co}_{15} \mathrm{Si}_{3}$ quasicrystal for ion incidence along a "random" direction and along the decagonal axis. For the channeling measurements presented in the subsequent figures two energy windows were set at about channel 175 and channel 245 in order to obtain the $\mathrm{Al} / \mathrm{Sj}$ - and $\mathrm{Co} / \mathrm{Cu}$-yield, respectively.

the spectra as indicated in fig. 1. The figure further shows that the backscattering rate for ion incidence along the decagonal axis is strongly suppressed due to axial channeling along this axis. This can be seen more clearly in fig. 2 where the $\mathrm{Co} / \mathrm{Cu}$ - and the $\mathrm{Al} / \mathrm{Si}$ scattering rates are plotted versus the angle of ion incidence. Both yield profiles show pronounced channeling minima with minimum yields $\chi_{\min }$ of 0.13 and 0.25 , and angular half-widths $\psi_{1 / 2}$ (HWHM) of $0.51^{\circ}$ and $0.37^{\circ}$, respectively.

One is tempted to derive from the angular halfwidths of the profiles structural parameters of the quasicrystal using Barrett's formula for ion channeling in periodic, monatomic structures [10]. With the energy of the ${ }^{4} \mathrm{He}^{+}$-ion beam of $2 \mathrm{MeV}$ as used in the experiments and a rms thermal amplitude of the lattice

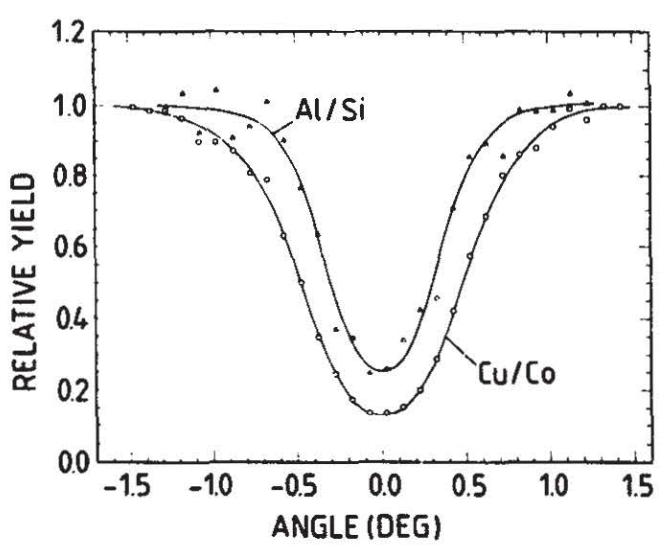

Fig. 2. Rutherford backscattering profiles as obtained from an angular scan through the decagonal axis of a $T$-phase $\mathrm{Al}_{62} \mathrm{Cu}_{20} \mathrm{Co}_{15} \mathrm{Si}_{3}$ quasicrystal. The lines are drawn to guide the eye. 
atoms of $7 \mathrm{pm}$, one obtains for the $\mathrm{Co} / \mathrm{Cu}$-scattering a mean atomic charge per unit string length, which is the decisive quantity for axial ion channeling, of $\left\langle Z_{2} / d\right\rangle=$ $41.4 \mathrm{~nm}^{-1}$. With a mean atomic charge of the atoms of the quasicrystal of $\left\langle Z_{2}\right\rangle=18.3$ one then finds an interatomic distance of $d=0.44 \mathrm{~nm}$ which is close to the values found in other investigations, which are in the range $0.408-0.428 \mathrm{~nm}$ (cf. e.g. ref. [6]). Channeling computer simulations based on the structure model derived by Steurer and Kuo [6] for this system (see also below, figs. 7 and 8) show that almost full agreement is obtained between calculated and experimental angular half-widths, when a proper treatment is adopted. These calculations also show that the difference between the $\mathrm{Co} / \mathrm{Cu}$ and $\mathrm{Al} / \mathrm{Si}$ profiles is primarily due to the different thermal vibration amplitudes of the atoms.

The existence of axial channeling along the decagonal axis is, however, nothing spectacular, since T-phase quasicrystals are periodic along this axis with the lattice atoms forming strings of a fixed interatomic distance $d$. Thus axial channeling along these strings should exist; only the arrangement of the strings in the transverse plane and the shapes of the channels formed by these strings are quasiperiodic. Nevertheless, the relatively deep minimum in the $\mathrm{Cu} / \mathrm{Co}$-scattering of $\chi_{\min }=0.13$ indicates, that the quasicrystal is of relatively good quality.

In a further series of experiments, the planar structure of this quasicrystal was examined. Fig. 3 shows the $\mathrm{Co} / \mathrm{Cu}$-yield as obtained from various angular scans in the (angular) neighbourhood of the decagonal axis. In these scans the quasicrystal was rotated with respect to the ion beam about a vertical axis (angle of rotation: $\theta_{x}$ ) at fixed tilt angles $\theta_{y}$ of the sample (rotation about a horizontal axis) and the scattering yield monitored as a function of $\theta_{x}$. Besides the deep minimum observed for the scan through the decagonal axis, several shallow minima are seen in the other scans. They are due to channeling in planes which have the decagonal axis in common. In fig. 4 the angular positions of the minima are plotted in a $\theta_{x}, \theta_{y}$-graph which provides the orientation diagram of the quasicrystal and is commonly used for crystal orientation purposes in ion beam experiments [11]. As the figure shows, these minima mark planes which have the decagonal axis in common; the stronger planes, i.e. which show the deeper minima (indicated by thick lines in fig. 4) meet at angles of $36^{\circ}$ with each other, thus proving the decagonal symmetry of the quasicrystal, while a set of weaker planes (thin lines) is found halfway between the main planes.

The existence of such planes and the observation of ion channeling in the channels formed by these planes is by no means trivial, since it requires the existence of linear atomic arrangements in the quasiperiodic layers of the crystal of (locally) decagonal symmetry which -

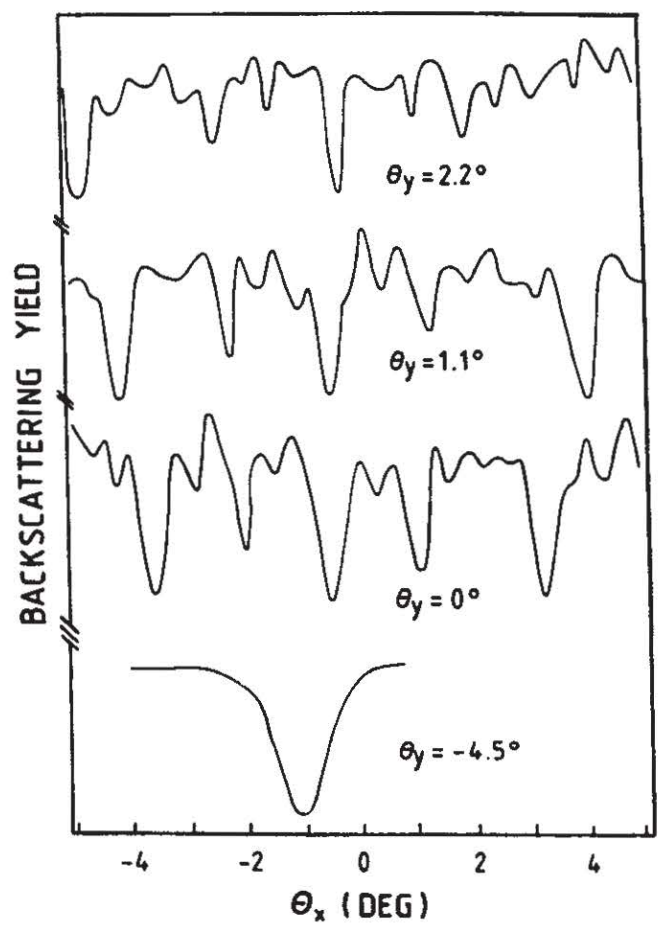

Fig. 3. Rutherford backscattering yield profiles $(\mathrm{Co} / \mathrm{Cu}$ scattering) as obtained from angular scans in the (angular) neighborhood of the decagonal axis of a T-phase $\mathrm{Al}_{62} \mathrm{Cu}_{20} \mathrm{Co}_{15} \mathrm{Si}_{3}$ quasicrystal. During the scans (rotation about a vertical axis, angle $\theta_{x}$ ) the quasicrystal was kept at various fixed tilting positions $\theta_{y}$. Channeling in the decagonal axial channel and in various planar channels is clearly visible.

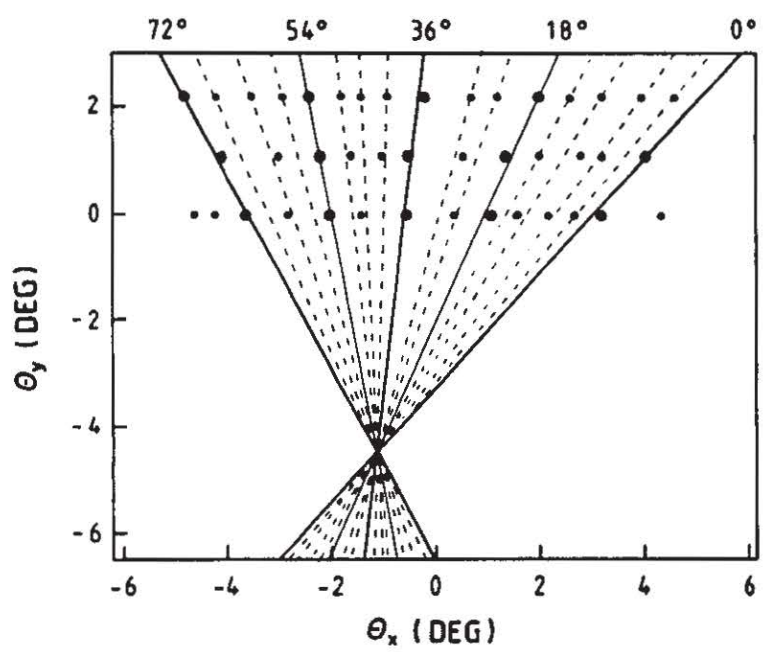

Fig. 4. Orientation diagram of a T-phase quasicrystal as obtained from the channeling results of fig. 3 . Besides the decagonal axis at $\theta_{x}=-1.1^{\circ}, \theta_{y}=-4.5^{\circ}$ various strong, weaker and weak planes (thick, thin and dashed lines, respectively) are indicated which show the tenfold symmetry of the quasicrystal. The angular positions of the weak planes are listed in table 1 (the angular positions of the ax:al channeling dips shown here and in fig. 2 differ due to rescaling.) 
Table 1

Comparison of the angles included by linear arrangements of vertices in a two-dimensional Penrose grid and angles between planes parallel to the decagonal axis (counted from the main planes) of a T-phase quasicrystal as derived from the present channeling measurements

\begin{tabular}{ll}
\hline Penrose $\llbracket$ deg] & Channeling [deg] \\
\hline 0 & 0 \\
2.491 & 2.5 \\
4.386 & 4.1 \\
5.554 & 5.6 \\
6.645 & - \\
7.563 & $7.8,7.5$ \\
9.732 & 9.7 \\
10.925 & - \\
13.614 & 13.9 \\
15.017 & 15.0 \\
15.510 & 15.5 \\
18 & 18.0 \\
\hline
\end{tabular}

stacked together periodically - form the planes observed in the channeling experiment. Thus, the observation of channeling in these planes may be considered as an independent proof of the quasicrystalline structure of this crystal; to a certain extent this proof is more straightforward than that by diffraction experiments, since ion channeling directly images the real structure of the crystal.

Besides the pronounced dips arising from axial channeling along the decagonal axis and in the system of planar channels discussed above, one observes also smaller dips in the yield profiles of fig. 3 . They are due to channeling in crystal planes which apparently are less densely occupied by lattice atoms and, therefore, exert only weaker steering forces on the motion of channeled ions. The angular positions of these planes are included in the orientation diagram of fig. 4 and marked by dashed lines. The azimuthal angles of the planes (counted from the main planes), as derived from this figure and from other experiments, are listed in table 1 together with angles obtained from the evaluation of corresponding linear arrangements of vertices in a two-dimensional Penrose pattern. As the table shows, there is close agreement between these values, once more proving the quasicrystalline nature of the crystal.

The folluwing parts of the paper deal with the question of how channeling may be used to provide information about the decoration of the quasicrystalline structure by the different atom species of the sample, information which is not obtained easily by other methods. Figs. 5 and 6 show angular scans through the main planes (at $0^{\circ}$ and $36^{\circ}$ ) and the shallower planes (at $18^{\circ}$ ) for both $\mathrm{Co} / \mathrm{Cu}-$ and $\mathrm{Al} / \mathrm{Si}$ scattering. The yield profiles of the main planes (fig. 5)

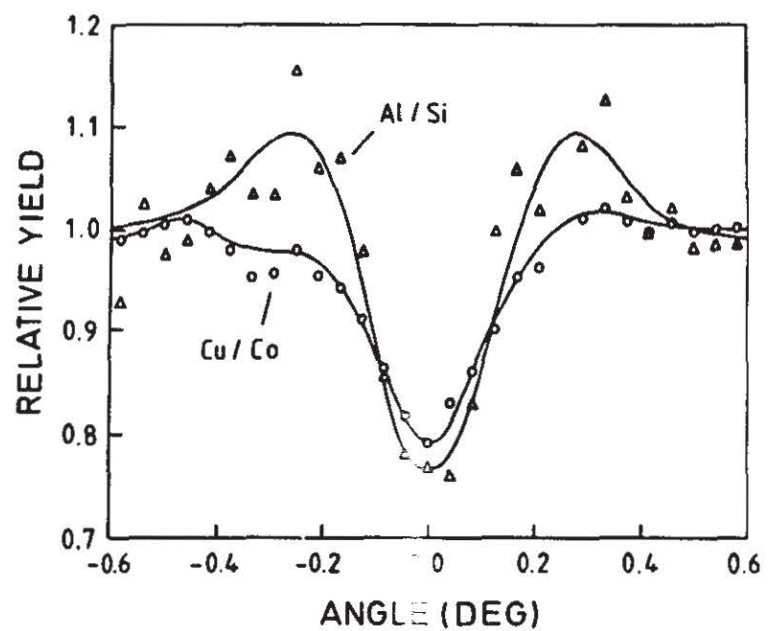

Fig. 5. Backscattering profiles from an angular scan across the main planar system parallel to the decagonal axis of an

$\mathrm{Al}_{62} \mathrm{Cu}_{20} \mathrm{Co}_{15} \mathrm{Si}_{3}$ T-phase quasicrystal (see also fig. 4).

are about equal in angular half-width and minimum yield. This indicates that all atom species are well aligned on these planes; only the high shoulders in the $\mathrm{Al} / \mathrm{Si}$-yield are indicative of small displacements of at least part of the $\mathrm{Al} / \mathrm{Si}$-atoms from the planes. In contrast, the yield profiles of the shallower planes (fig. 6) differ by about a factor of 2 . Also here pronounced shoulders are observed in the $\mathrm{Al} / \mathrm{Si}$-yield.

The results of figs. 2, 5 and 6 are to be compared with the results of channeling computer simulations which are shown in figs. 7 and 8 . The principles of such calculations have been described briefly in ref. [7]; a more detailed description of channeling computer simulations in ordinary crystais may be found in refs. $[10,12]$. The calculations have been performed on a

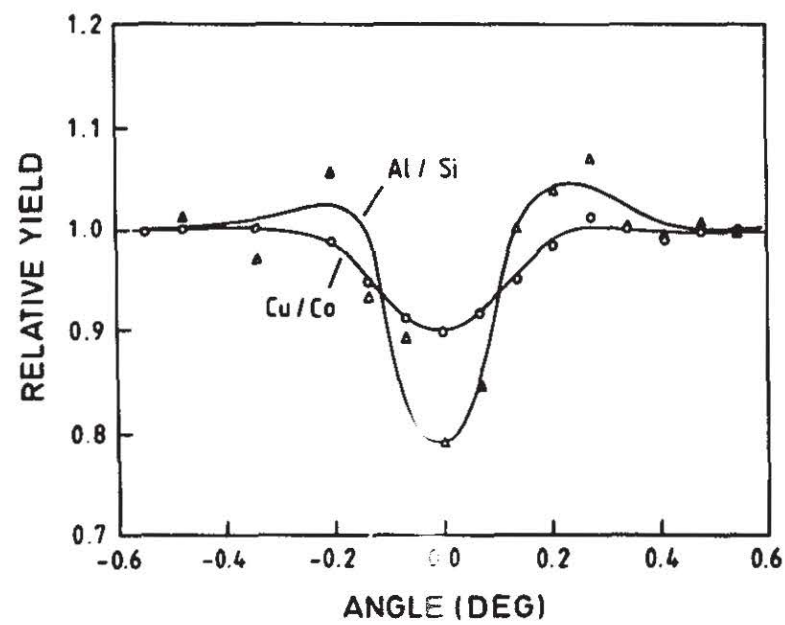

Fig. 6. Backscattering profiles from an angular scan across the plane system halfway between the main planar system of an $\mathrm{Al}_{62} \mathrm{Cu}_{20} \mathrm{Co}_{15} \mathrm{Si}_{3} \mathrm{~T}$-phase quasicrystal (see also fig. 4). 


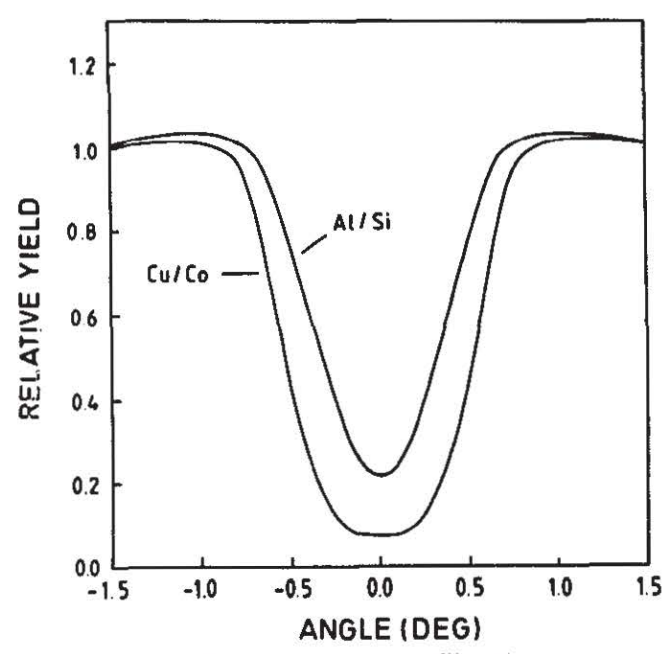

Fig. 7. Channeling backscattering profile of the decagonal axis of an $\mathrm{Al}_{65} \mathrm{Cu}_{20} \mathrm{Co}_{15} \mathrm{~T}$-phase quasicrystal as calculated in channeling computer simulations on a model structure proposed by Steurer and Kuo [6].

model T-phase quasicrystal proposed by Steurer and Kuo [6]. It has been derived from X-ray diffraction data on $\mathrm{T}$-phase $\mathrm{Al}_{65} \mathrm{Cu}_{20} \mathrm{Co}_{15}$ quasicrystals by highdimensional embedding technique and five-dimensional Patterson synthesis (see e.g. ref. [5]). For further details on the structure model as well as on the channeling computer simulations in this structure, we refer to a forthcoming paper [15].

Fig. 7 shows backscattering yield profiles as calculated for an angular scan across the decagonal axis of the quasicrystal. Both yield profiles, which have been calculated for rms thermal amplitudes of 7 and $10 \mathrm{pm}$

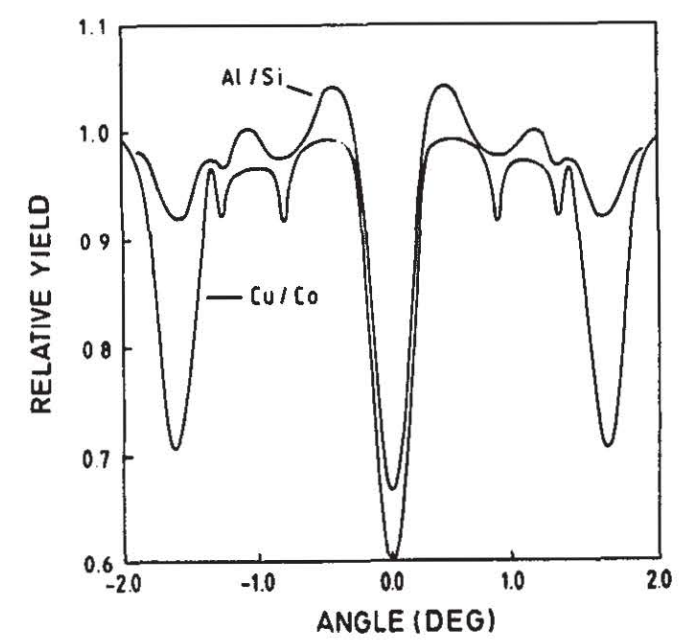

Fig. 8. Channeling backscattering profiles for a T-phase $\mathrm{Al}_{65} \mathrm{Cu}_{20} \mathrm{Co}_{15}$ quasicrystal as calculated in channeling computer simulations on a model structure proposed by Steurer and Kuo [6]. Shown is an angular scan across the main planar system (at the centre) and various weaker planes. of the $\mathrm{Co} / \mathrm{Cu}$ - and $\mathrm{Al} / \mathrm{Si}$-atoms, respectively, are in very reasonable agreement with the measurements shown in fig. 2. For channeling in the main planes (figs. 5 and 8, central part) both $\mathrm{Co} / \mathrm{Cu}$ - and $\mathrm{Al} /$ Si-profiles exhibit almost identical shapes in the calculated as well as in the experimental profiles. However, the calculated profiles are somewhat deeper which may be due to the rather shallow ion penetration depth used in the calculations. We would like to draw the reader's attention to the fact that even the high shoulders in the $\mathrm{Al} / \mathrm{Si}$-yield are reproduced by the calculations.

The situation is different for the shallower planes at $18^{\circ}$ (figs. 6 and 8, left and right part of the profiles). While in the experimental yield profiles the $\mathrm{Al} / \mathrm{Si}$-profile exhibits about twice the depth of the $\mathrm{Co} / \mathrm{Cu}$-profiles, this is reversed in the calculated profiles: the depth of the $\mathrm{Al} / \mathrm{Si}$-profiles is only about one quarter of that of the $\mathrm{Co} / \mathrm{Cu}$-profiles. This is too serious a discrepancy to be due to experimental inaccuracies or different conditions of calculation and experiment. It does seem to indicate differences between the model proposed by Steurer and Kuo and the structure of the quasicrystal used in the experiments. This fact will be studied in detail in a subsequent investigation.

Up to this point no distinction was possible between Co- and Cu-atoms. In order to obtain information on the details of the decoration of the quasicrystal by these atoms, ion channeling was combined with PIXE. For this purpose a $3 \mathrm{MeV}{ }^{4} \mathrm{He}^{+}$-beam was used. $\mathrm{A}$ typical X-ray spectrum, as obtained in these measurements, is shown in fig. 9. For the channeling measurements energy windows were set on the $A l K_{\alpha}$, the $\mathrm{CoK}_{\alpha}$ - and the $\mathrm{Cu} \mathrm{K}_{\alpha}$-peak as indicated in fig. 9. Figs. 10 and 11 show the results for angular scans across the decagonal axis and the main plane system. For the sake

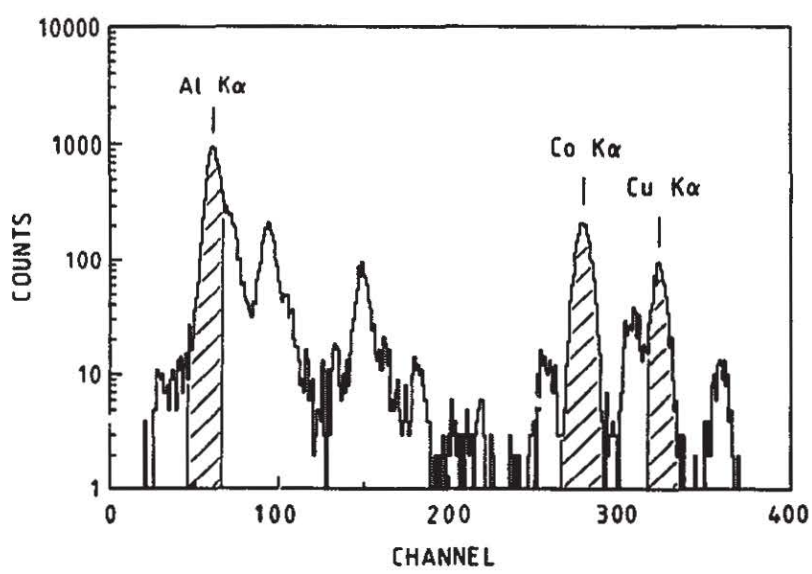

Fig. 9. PIXE spectrum as obtained from a T-phase $\mathrm{Al}_{62} \mathrm{Cu}_{20} \mathrm{Co}_{15} \mathrm{Si}_{3}$ quasicrystal with $3 \mathrm{MeV}{ }^{4} \mathrm{He}^{+}$-ions. The windows set on the different $\mathrm{X}$-ray peaks were used for the channeling measurements presented in the subsequent figures. 


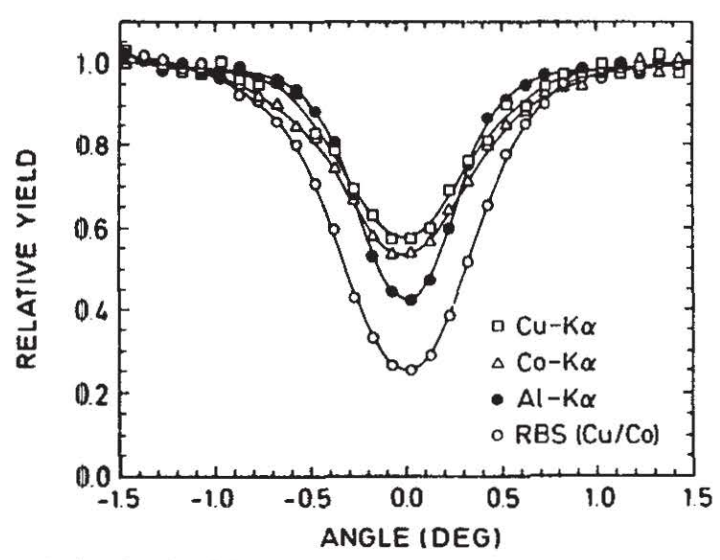

Fig. 10. Rutherford backscattering and $X$-ray profiles as obtained from an angular scan across the decagonal axis of a T-phase $\mathrm{Al}_{62} \mathrm{Cu}_{20} \mathrm{Co}_{15} \mathrm{Si}_{3}$ quasicrystal. The lines are drawn to guide the eye.

of comparison the $\mathrm{Co} / \mathrm{Cu}$-backscattering yield profiles are shown. In both cases the $\mathrm{AJ} \mathrm{K}_{\mathrm{a}}$-yield profile shows the lowest minimum yield, since only X-rays from the near surface region of the quasicrystal are counted due to the high absorption coefficient of the soft $\mathrm{AlK} \mathrm{K}_{\alpha}-\mathrm{X}$ rays. For channeling along the decagonal axis (fig. 10), the $\mathrm{Co}$ and $\mathrm{Cu} \mathrm{X}$-ray profiles are nearly identical. In contrast, for channeling along the main planes (fig. 11), the depth of the Co X-ray profile is about twice that of the $\mathrm{Cu} \mathrm{X}$-ray profile. This information has to be incorporated into the model of the quasicrystal; it should be noted that the evaluation of $\mathrm{X}$-ray diffraction data as performed by Steurer and Kuo does not allow one to distinguish between $\mathrm{Co}$ - and $\mathrm{Cu}$-atoms.

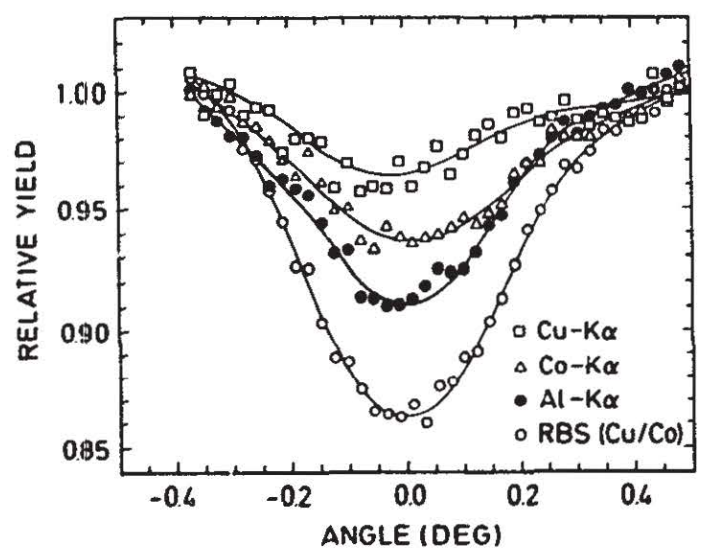

Fig. 11. Rutherford backscattering and $X$-ray profiles from an angular scan acrosis the main planar system of a $T$-phase $\mathrm{Al}_{62} \mathrm{Cu}_{20} \mathrm{Co}_{15} \mathrm{Si}_{3}$ quasicrystal.

\section{Conciusions}

It has been the aim of this investigation to show that ion channcling in quasicrystals is possible, as was proposed by Kupke et al. [7] in a theoretical study of icosahedral quasicrystals. From this study of T-phase $\mathrm{Al}_{62} \mathrm{Cu}_{20} \mathrm{Co}_{15} \mathrm{Si}_{3}$ quasicrystals which are periodic along the decagonal axis, but quasipcriodic in the layers normal to this axis, three major findings have been made. (i) There is pronounced axial channeling along the decagonal axis. (ii) There is planar channeling in various planes which have the decagonal axis in common and exhibit tenfold symmetry around this axis. Thus the findings prove the existence of such planar arrangements of lattice atoms in a T-phase quasicrystal parallel to the decagonal axis. (iii) The angles between these planes correspond to the angles between the linear arrangements of vertices found in a Penrose grid, which demonstrates the close relation between T-phase quasicrystals and Penrose pattern. Since channeling measurements sample a macroscopic part of a crystal and give a direct picture of the crystal structure - in contrast to e.g. electron transmission microscopy - , the present results strongly support the concept of quasicrystals.

It has also been shown that ion channeling is very well able to provide structural information on the individual atom species present in a sample, i.c. on the decoration of a structure, information which is hardly obtainable by other methods (with possibly the exception of neutron scattering). Perhaps the most useful application of ion channeling will be its use for the localization of foreign atoms and the study of lattice imperfections, subjects which have been investigated by this method with great success on ordinary crystals in the past.

\section{Acknowledgements}

The authors would like to thank Dr. J. Diehl, W. Decker, Dr. H. Stoll, and the technical staff of the Pelletron accelerator at the Max-Planck-Institut für Metallforschung, Stuttgart, for their kind cooperation.

\section{References}

[1] L. Pauling, Phys. Rev. Lett. 58 (1987) 365.

[2] P.W. Stephen and A.T. Goldman, Phys. Rev. Lett. 56 (1986) 1168.

[3] P. Kramer and R. Neri, Acta Crystallogr. A40 (1984) 580.

[4] R. Penrose, Bull. Inst. Math. Appl. 10 (1974) 266.

[5] W. Steurer, Z. Kristallogr. 190 (1990) 179.

[6] W. Steurer and K.H. Kuo, Philos. Mag. Lett. 62 (1990) 175. 
[7] T. Kupke, U. Peschke, H.D. Carstanjen and H.R. Trebin, Phys. Rev. B43 (1991) 13758.

[8] H.D. Carstanjen, R.M. Emrick, R. Grunwald, D. Plachke and R. Wittmann, submitted to Phys. Rev. Lett.

[9] L.X. He, K. Wu, M. Meng and K.H. Kuo, Philos. Mag. Lett. 61 (1090) 15.

[10] J.H. Barrett, Phys. Rev. B3 (1971) 1527.

[11] R.S. Nelson, Philos. Mag. 15 (1967) 845.

[12] H.D. Carstanjen and R. Sizmann, Radiat. Eff. 12 (1972) 211.
[13] R. Grunwald, R.M. Emrick, D. Plachke, R. Wittmann und H.D. Carstanjen, Verhandl. DPG (VI) 26 (1991) 1288.

[14] E.H. du Marchie van Voorthuysen, P.J.M. Smulders, R.D. Werkman, J.L. de Boer and S. van Smaalen, Proc. 10th Int. Conf. on Jon Beam Analysis, Eindhoven, Netherlands, 1991, Nucl. Instr. and Meth. B64 (1992) 261.

[15] H.D. Carstanjen, T. Kupke, D. Plachke and H.-R. Trebin, to be published. 